\title{
Supervivencia de larvas de cachama blanca, Piaractus brachypomus Cuvier 1818, sometidas a cambios experimentales de temperatura
}

\section{Sobrevivência das larvas de pirapitinga, Piaractus brachypomus Cuvier 1818, sob mudanças de temperatura experimentais}

\section{Cachama blanca larvae's survival, Piaractus brachypomus Cuvier 1818, in experimental temperature changes}

\section{Luis F. Collazos-Lasso ${ }^{1 *}$; Mariana C. Gutiérrez-Espinosa ${ }^{2 *}$; Luis F. Restrepo-Betancur ${ }^{3}$}

\author{
Ing. producción Acuícola, MSc, Docente Instituto de Acuicultura de la Universidad de los Llanos \\ 2 Zootecnista, MSc, Docente Instituto de Acuicultura de la Universidad de los Llanos \\ 3 Estadistico, Facultad de Ciencias Agrarias, Universidad de Antioquia, Medellín, Colombia \\ Instituto de Acuicultura de la Universidad de los Llanos. Grupo de Investigación IALL Villavicencio, Colombia \\ Email: Ifclasso@yahoo.com
}

Recibido: octubre 30 de $2014 \quad$ Aceptado: noviembre 28 de 2014

\begin{abstract}
Resumen
La cachama blanca es la especie íctica nativa de mayor producción en Colombia, sin embargo la obtención de semilla aún es limitada, en principio por la estacionalidad reproductiva de la especie y la baja supervivencia en larvicultura, que consiste en sembrar larvas en estanques abonados donde permanecen hasta alevinos, previa aclimatación. El objetivo del presente estudio fue establecer el rango de confort y los tiempos de respuesta de ajuste a los cambios de temperatura experimental, teniendo como indicador la supervivencia de larvas de cachama. Los ensayos se realizaron en el Instituto de Acuicultura de la Universidad de los Llanos (IALL). Se utilizaron 1020 larvas de cachama, de 168 horas pos-eclosión, obtenidas mediante reproducción inducida con extracto de hipófisis de carpa, alimentadas a las $72 \mathrm{HPE}(\mathrm{C} / 6 \mathrm{~h})$ con náuplios de artemia. La calidad del agua en incubadora (tipo Woynarovich) fue: temperatura $27 \pm 0.4^{\circ} \mathrm{C} ; \mathrm{pH} 6,5 \pm 0.2 ; \mathrm{O}_{2} 6 \pm 0.4 \mathrm{mg} / \mathrm{l} ; \mathrm{dureza} 30 \pm 5$ mg. Las larvas fueron colectadas y contenidas en seis beakers ( $40 \mathrm{ml}$ de agua) dispuestos en una incubadora térmica (Rev. Scien. Mod. RS-IF-201) con previa calibración que garantizaba la temperatura a experimentar. Cada temperatura fue un tratamiento y cada beaker una réplica (15 larvas). El conteo de la supervivencia larvaria de cada una de las réplicas se realizó cada cinco minutos durante una hora. El rango de confort se estableció entre $22-29^{\circ} \mathrm{C}$, el rango de estrés inferior entre $18-22^{\circ} \mathrm{C}$, el rango de estrés superior entre $29-32^{\circ} \mathrm{C}$ y el rango crítico inferior a partir de $18^{\circ} \mathrm{C}$. La temperatura letal 50 se estableció en $9,5^{\circ} \mathrm{C}$ y la temperatura letal inferior en $6^{\circ} \mathrm{C}$ (Para las temperaturas que se presentan entre rangos no se encontraron diferencias estadísticas significativas, en relación con la supervivencia/Alpha=0,05).
\end{abstract}

Palabras claves: Peces, carácidos, aclimatación, estrés, temperatura confort.

\begin{abstract}
Cachama blanca is the native fish species with the highest production in Colombia, however obtaining seed is still limited, mainly because of the reproductive seasonality of the species and the survivals low hatchery, which consists on planting
\end{abstract}


larvae in ponds where subscribers remain to fry, previous acclimatization. The aim of this study was to establish the range of comfort and response times adjusting to changes in experimental temperature, taking the survival of cachama blanca larvae as an indicator. Trials were conducted at the Instituto de Acuicultura de la Universidad de los Llanos (IALL). 1020 cachama blanca larvae of 168 post-hatching hours were used, obtained by induced breeding carp pituitary extract, fed with artemia at $72 \mathrm{HPE}(\mathrm{C} / 6 \mathrm{~h})$. Water quality incubator (Woynarovich type) was: temperature $27 \pm 0.4{ }^{\circ} \mathrm{C} ; \mathrm{pH} 6.5 \pm 0.2 ; \mathrm{O} 26 \pm 0.4 \mathrm{mg}$ / I; hardness $30 \pm 5 \mathrm{mg}$; the larvae were collected and contained in six beakers (40 ml water) arranged in a thermal incubator (Rev. Scien. Mod. RS-IF-201) with previous calibration temperature guaranteeing experience. Each temperature was treated and each beaker a replica (15 larvae). Counting the larval survival of each of the replicas it was performed every five minutes for one hour; Results: the comfort range was established between $22-29{ }^{\circ} \mathrm{C}$, lower stress range between $18-22{ }^{\circ} \mathrm{C}$, the range between $29-32{ }^{\circ} \mathrm{C}$ higher stress, lower from $18^{\circ} \mathrm{C}$ critical range, lethal temperature 50 was established in $9,5{ }^{\circ} \mathrm{C}$ and the lethal temperature below $6{ }^{\circ} \mathrm{C}$ (for temperatures between ranges presented no statistically significant differences were found in relation to the survival/Alpha $=0,05$ )

Keywords: Fish, characins, acclimatization, stress, temperature comfort.

\section{Resumo}

O pirapitinga é a espécie de peixe nativa com maior produção na Colômbia, no entanto obtenção de sementes ainda é limitado substancialmente pela sazonalidade reprodutiva das espécies e as sobrevivências baixo larvicultura, que consiste no plantio de larvas em lagoas onde os assinantes permanecer para alevim, aclimatação prévia. O objetivo deste estudo foi estabelecer o rango de conforto e tempos de resposta ajustar às mudanças de temperatura experimental, tendo como indicador a sobrevivência de larvas de pirapitinga. Os testes foram realizados no Instituto de Aquicultura de la Universidad de los Llanos (IALL), 1020 larvas de pirapitinga, 168 horas pós-eclosão, obtido por reprodução induzida extrato de hipófise de carpa, alimentados a $72 \mathrm{HPE}(\mathrm{C} / 6 \mathrm{~h})$ com Artemia, qualidade da água na incubadora (tipo WOYNAROVICH) foi: temperatura de $27 \pm 0,4^{\circ} \mathrm{C} ; \mathrm{pH} 6,5 \pm 0,2 ; \mathrm{O} 26 \pm 0,4 \mathrm{mg} / \mathrm{l}$; dureza de $30 \pm 5 \mathrm{mg}$; as larvas foram compilados e armazenados nos seis taças (40 ml de água) dispostas em uma incubadora térmica (Rev. Ciência. Mod. RS-IF-201) com temperatura de calibração anterior garantindo experiência. Cada temperatura e tratou-se cada proveta de uma réplica (15 larvas). Contando a sobrevivência das larvas de cada das réplicas foi realizada a cada cinco minutos durante uma hora; resultados: a faixa de conforto foi estabelecida entre $22-29{ }^{\circ} \mathrm{C}$, a faixa de estresse menor entre $18-22{ }^{\circ} \mathrm{C}$, a faixa de estresse maior entre $29-32{ }^{\circ} \mathrm{C}$ estresse, menor de $18{ }^{\circ} \mathrm{C}$ faixa crítica, temperatura letal 50 foi estabelecido em $9,5{ }^{\circ} \mathrm{C}$ e a temperatura letal abaixo de $6{ }^{\circ} \mathrm{C}$ (para temperaturas entre os intervalos não apresentaram diferenças estatisticamente significativas foram encontradas, em relação à sobrevivência/Alpha=0,05).

Palavras-chave: Peixe, caracídeos, climatização, estresse, conforto térmico.

\section{Introducción}

La acuicultura se ha ubicado como uno de los sectores productivos agrarios de mayor crecimiento en la economía mundial, con un incremento en la tasa media anual del 8.6\% desde 1980 (FAO, 2014). Para Colombia, este aumento ha sido del $12 \%$ desde 1990. Para el 2011 se reportó una producción de 74.270 toneladas provenientes de la piscicultura, especialmente de tilapia $(65,21 \%)$, cachama blanca $(21,44 \%)$ y trucha (7,58\%) (AUNAP, 2013).

Teniendo en cuenta lo anterior, la cachama blanca, Piaractus brachypomus, es la especie íctica nativa con mayor volumen de producción y, por ende, la más cultivada en Colombia, debido en gran medida a que se puede reproducir en cautiverio, es de hábitos alimenticios omnívoros, presenta rápido crecimiento, una alta eficiencia de conversión de alimento, y es resistente al manejo y a las enfermedades (Vásquez y Zacarías, 1996; Vásquez, et al., 2002; Navarro, et al., 2004; Landinez y Mojica, 2005; Peñuela, et al., 2007; Vásquez, 2013).

No obstante, la producción de larvas aún es limitada, principalmente por la estacionalidad reproductiva propia de la especie y las bajas supervivencias en la larvicultura. De este último aspecto, Atencio (2001) menciona que la adaptación del sistema Húngaro es el más usado en la producción de larvas, el cual consiste en sembrarlas en estanques abonados donde permanecen hasta convertirse en alevinos.

Dentro de las diferentes etapas o actividades para la producción en confinamiento de una especie íctica, la larvicultura es de vital importancia, puesto que el éxito comercial de un cultivo de peces depende en gran medida de la producción controlada de un número suficiente de alevinos de la especie a cultivar (Atencio, et al, 2003; Lazo, 2000). En este sentido, la supervivencia de las larvas es un aspecto primordial que está directamente relacionado con la plena satisfacción de sus requerimientos. En consecuencia, los resultados pueden ser favorables si la alimentación es la adecuada y la calidad del agua, está en los rangos de confort para la especie cultivada (Atencio, et al., 2003b; David, et al., 2011, Collazos y Arias, 2009, Chippari y Baldissertto, 2000). 
Durante la larvicultura, se realiza una actividad que es considerada fundamental para garantizar la supervivencia de las larvas al momento de la siembra en estanques, denominada aclimatación. Se define como la acción de equilibrar la temperatura del agua de las bolsas en las que son transportadas las larvas y la temperatura de los estanques durante la siembra.

Ante estas posibles variaciones de temperatura, los peces han desarrollado diferentes mecanismos para responder o contrarrestar estos cambios. Sin embargo, en muchas especies tales mecanismos son limitados y solo son eficientes dentro de variaciones de temperatura pequeñas $\left(3-5^{\circ} \mathrm{C}\right)$, en lapsos relativamente largos (por lo menos 12 horas) (Parker y Davis 1981; Moyle y Cech, 2004). Los ajustes que los peces realizan a variaciones pequeñas de temperatura, que se podría decir son de tipo instantáneo y que no causan cambios importantes en el comportamiento del animal, constituyen lo que se ha denominado rango de confort, definiéndose entonces este, para un determinado parámetro, como el intervalo en el cual el individuo no sufre cambios aparentes de actividad, desempeño o comportamiento (Osse y Van Den Boogaart, 1995). El objetivo de este trabajo fue establecer el rango de confort y los tiempos de respuesta de ajuste a los cambios de temperatura, teniendo como indicador la supervivencia de las larvas de 168 horas pos-eclosión de cachama blanca.

\section{Materiales y métodos}

Los ensayos se realizaron en el Instituto de Acuicultura de la Universidad de los Llanos - IALL, Villavicencio (Meta - Colombia), ubicado en el Km 4 Vía Puerto López. Vereda Barcelona. Para este estudio se utilizaron 1020 larvas de cachama blanca, Piaractus brachypomus, de 168 horas pos-eclosión (HPE), obtenidas mediante reproducción inducida con extracto de hipófisis de carpa (5,5 mg/Kg 9 en 2 dosis y 4,4 mg/Kg $\widehat{\jmath}$ dosis única). Las larvas obtenidas recibieron una primera alimentación con naúplios de Artemia salina, cada seis horas a partir de las 72 hpe. Se monitorearon los parámetros de calidad de agua (temperatura $27 \pm 0.4{ }^{\circ} \mathrm{C}$; $\mathrm{pH} 6,5 \pm 0.2 ; \mathrm{O}_{2} 6 \pm 0.4 \mathrm{mg} / \mathrm{l}$; dureza $30 \pm 5 \mathrm{mg}$ ) y se mantuvieron en incubadora Woynarovich hasta iniciar los ensayos.

Para la medición de la temperatura se utilizó un termómetro digital HANNA INSTRUMENTS con rango de operación $0-100{ }^{\circ} \mathrm{C}$ y precisión de $0,1{ }^{\circ} \mathrm{C}$.; el oxígeno disuelto con un oxímetro digital (Sonda multiparamétrica YSI 556), con rango entre $0-50 \mathrm{mg} / \mathrm{L}$ y resolución de 0,01 mg/L, previamente calibrado según las instrucciones del fabricante; el pH (potencial hidroge- niónico), mediante un pH-metro digital portátil, modelo CORNING, calibrado con soluciones buffer $\mathrm{pH} 4.01$ y 7.01; la dureza, con un kit para análisis de dureza. MOL LABS Ltda.

Para evaluar la supervivencia con respecto a la temperatura, se tomaron muestras de las larvas (168 HPE) colectándolas de una incubadora tipo Woynarovich en un beaker de 500 ml, el cual era llevado en una caja de icopor, con el fin de mantener la temperatura del agua. Esta muestra se dividió en cuatro submuestras (réplicas), y se colocaron en beakers de $100 \mathrm{ml}$ (40 ml útiles). Esta operación se realizó para cada una de las temperaturas a evaluar (27 tratamientos). Los beakers fueron localizados previamente en una incubadora térmica (Rev. Scien. Mod. RS-IF-201) durante una hora, para calibración y mantenimiento constante de la temperatura a experimentar. Cabe anotar que el agua utilizada provenía de la incubadora donde permanecían las larvas, controlando de esta manera las posibles variables (condiciones fisicoquímicas del agua) que podrían intervenir en el ensayo. Cada réplica estaba conformada por 15 larvas. El conteo de la supervivencia larvaria se realizó cada cinco minutos durante una hora.

\section{Análisis estadístico}

Se empleó el Modelo Lineal General (GLM) de 1 y 2 vías y se suplemento con la prueba de contraste de Tukey con base en un 95\% de confiabilidad. Convalidando los supuestos adscritos con cada modelo de clasificación experimental. Adicionalmente se efectuaron análisis particulares para cada tiempo, con el fin de controlar si existía diferencia estadística en el efecto de temperatura. Finalmente se realizó análisis descriptivo exploratorio tridimensional para establecer promedio, desviación y coeficiente de variación. Se empleó el paquete estadístico SAS ver 8.2.

Diagrama de estructura por tiempo: $Y_{i j}: M+T_{i}+E_{j(i)}$

M: efecto promedio, $\mathrm{T}$ : efecto de la $\mathrm{T}^{\circ}, \mathrm{E}$ : error experimental

Diagrama análisis global: $Y_{i j}: M+T_{i}+T^{*}+E_{j(i)}$

M: efecto promedio, $\mathrm{T}$ : efecto de la $\mathrm{T}^{\circ}, \mathrm{E}$ : error experimental, $\mathrm{T}^{*}$ : efecto del tiempo

\section{Resultados}

En las figuras 1 y 2 se presentan los resultados obtenidos en la presente experimentación, en relación con la supervivencia de larvas de cachama blanca expuestas 


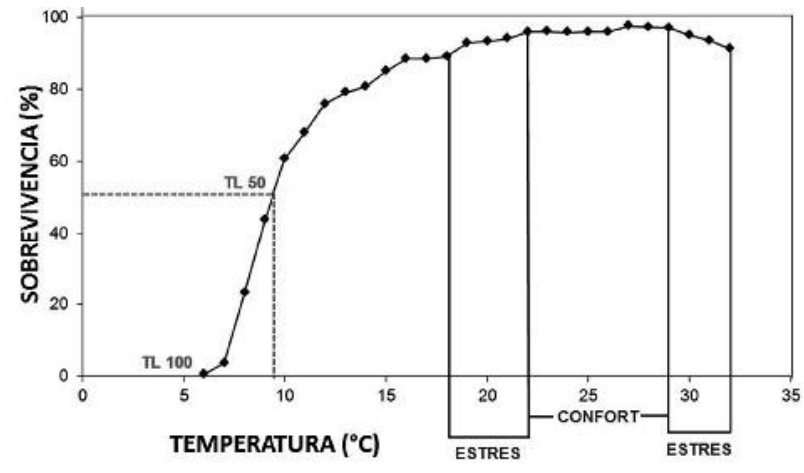

Figura1. Porcentaje de supervivencia de larvas de cachama blanca de 168 horas pos-eclosiòn, sometidas a cambios experimentales de temperatura; el rango de confort se estableció entre $22-29^{\circ} \mathrm{C}$, el rango de estrés inferior entre 18 $-22^{\circ} \mathrm{C}$, el rango de estrés superior entre $29-32^{\circ} \mathrm{C}$, el rango crítico inferior a partir de $18^{\mathrm{a}} \mathrm{C}$, la temperatura inferior letal 50 (TL50) en $9,5^{\circ} \mathrm{C}$ y la temperatura letal inferior (TL100) en $6^{\circ} \mathrm{C}$. (Para las temperaturas que se presentan entre rangos no se encontraron diferencias estadísticas significativas, en relación con los resultados de supervivencia / Alpha $=0.05$ ).

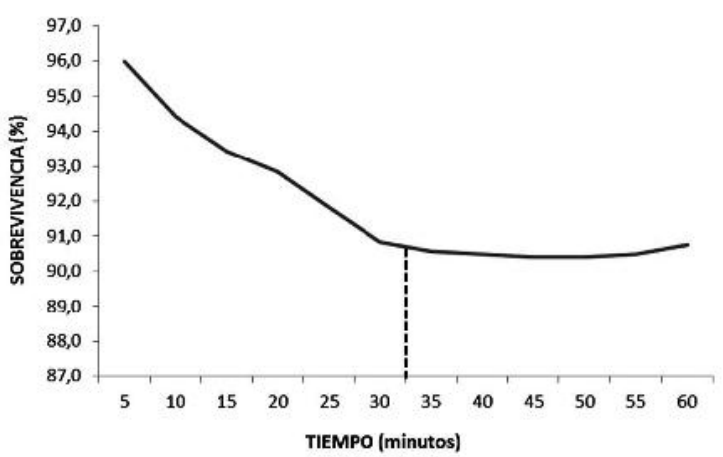

Figura 2. Tiempo de aclimatación ( $\left.\begin{array}{l}1 \\ 1\end{array}\right)$, de larvas de cachama blanca de 168 horas pos-eclosión, dentro del rango de confort establecido para temperatura.

a diferentes temperaturas y el tiempo de aclimatación a dichas variaciones.

\section{Discusión}

La temperatura es un factor ecológico importante por los efectos directos que tiene en la supervivencia de los peces (Chippari et al, 2000). Es por esta razón que dentro de las actividades de siembra de larvas es necesario establecer los rangos de temperatura y el tiempo en el que se debe hacer la aclimatación. Como consecuencia, disminuye la posible mortalidad por esta variable.

En estudios realizados por Arias y Vásquez (1988) relacionados con aspectos biológicos de la cachama blanca, se presentan monitoreos y registros de parámetros físico - químicos durante un año, de la parte alta del curso medio del río Meta en la Orinoquia Colombiana. En los resultados obtenidos en dos puntos, para el primero muestran una temperatura promedio es de $27,1 \pm 0,8{ }^{\circ} \mathrm{C}$, con unos máximos y mínimos de 28,7 ${ }^{\circ} \mathrm{C}$ en noviembre y 26,2 en marzo. En el segundo sitio registraron una temperatura promedio de $24 \pm 1,35^{\circ} \mathrm{C}$ con unos máximos y mínimos de $27^{\circ} \mathrm{C}$ en febrero y 23 ${ }^{\circ} \mathrm{C}$ en junio, respectivamente.

Teniendo en cuenta lo anterior, los mismos autores plantean que la maduración final se relaciona directamente con el inicio de la época invernal, que es cuando migran aguas arriba (característica propia de las especies reofilicas tropicales), en búsqueda de lugares y condiciones adecuadas para desovar; una vez los huevos son liberados en los afluentes altos de la cuenca, recorren largas distancias hasta que eclosionan e inician la fase larval. Bajo las anteriores premisas encontramos que en ambientes naturales, las larvas de cachama se encontraban en rangos de temperatura que concuerdan con la termorregulación comportamental confortable que mostraron las larvas de cachama expuestas a cambios experimentales de temperatura entre 22 y $29^{\circ} \mathrm{C}$ con sobrevivencias mayores al 95\%, como resultados del presente estudio.

En condiciones de cultivo, los registros de seguimiento de parámetros físico-químicos del agua, en un estudio relacionado con materia orgánica en estanques piscícolas, desarrollado en el departamento del Meta - Colombia, durante un año (dos ciclos de cultivo), registraron una temperatura promedio de $26{ }^{\circ} \mathrm{C}$ con picos máximos de $29{ }^{\circ} \mathrm{C}$ y minino de $23{ }^{\circ} \mathrm{C}$ (Yossa, et al., 2011).

En este sentido, se puede llegar a establecer que el rango de confort en temperatura encontrado de manera experimental para la cachama blanca, se enmarca en las fluctuaciones térmicas registradas en el agua de las piscícolas, y ambientes naturales de la cuenca del rio Meta.

Como lo plantea Chippari et al., (2000), las temperaturas inmediatamente por debajo y por encima del rango de confort, a las cuales se les ha denominado "rangos de estrés", presentan en las larvas una respuesta visible inmediata, tales como cambio de color y pérdida del eje de nado, condiciones similares a las expuestas por Parker y Davis (1981); en el presente estudio dichos rangos se establecieron entre $18-22^{\circ} \mathrm{C}$ (inferior) y entre 29 y $32^{\circ} \mathrm{C}$ como el rango de estrés superior, similar a lo reportado para la especie nativa Rhamdia sebae por Collazos y Arias (2007).

En trabajos realizados por Morales et al., (2014), concluyen que la supervivencia de la especie Poecilia re- 
ticulata disminuye a temperaturas inferiores a $10,3{ }^{\circ} \mathrm{C}$. Resultados similares se obtuvieron en larvas de cachama, donde se determinó que la temperatura letal 50 inferior fue a $9,5^{\circ} \mathrm{C}$ y la letal 100 a los $6{ }^{\circ} \mathrm{C}$.

\section{Conclusión}

El tiempo de aclimatación dentro del rango de confort para temperatura $\left(22-29{ }^{\circ} \mathrm{C}\right)$ es de 30 minutos, las larvas de cachama son más sensibles a estas variaciones que las larvas de otras especies tropicales, para la barbilla por ejemplo, se sugiere un tiempo de aclimatación mínimo de 10 minutos en rangos de temperatura entre 14 y $33{ }^{\circ} \mathrm{C}$ con supervivencias por encima del $80 \%$ (Collazos y Arias, 2007), posiblemente esto se debe a la limitada distribución geográfica de la especie estudiada.

Para larvas de cachama blanca, el rango de temperatura confort se estableció entre $22-29{ }^{\circ} \mathrm{C}$ y se hace necesario un período de aclimatación de treinta minutos.

\section{Referencias}

Arias CJA, Vásquez TW. 1988. Ampliación del conocimiento biológico de Colossoma sp. En ambientes naturales de la cuenca del río Meta. Informe de resultados Colciencias.

Atencio-García V. Producción de alevinos de especies nativas. Rev MVZ Córdoba. 2001; 6: 9-14.

Atencio GV, Kerguelen E, Wadnipar L, Narvaez A. Manejo de la primera alimentación del bocachico (Prochilodus magdalenae). Rev MVZ Córdoba. 2003; 8:254-260.

Atencio GV, Zaniboni FE, Pardo CS, Arias CA. In $\square$ uência da primeira alimentação na larvicultura e alevinagem do yamú Brycon siebenthalae. Acta Scientarium. 2003b;25(1):61-72.

AUNAP - Autoridad Nacional de Acuicultura y Pesca. 2013. Diagnóstico del estado de la acuicultura en Colombia. Bogotá, Colombia.

Chippari A, Gomes L, Baldisserotto B. Lethal temperatures for Rhamdia quelen larvae (Pimelodidae). Santa Maria, Brasil. Cien Rural. 2000;30(6):1069-1071.

Collazos LF, Arias JA.2007. Influencia de la temperatura en la supervivencia de larvas de Rhamdia sebae c.f. (Siluriformes heptapteridae). Orinoquia. 2007;11(1):56-62.

Collazos LF, Arias JA. Respuesta de larvas de Ancistrus triradiatus, a diferentes ofertas de primera alimentación. Orinoquia. 2009;13(2): 87-92.

David C, Lenis G, Castañeda G, Lopera A, Restrepo Lf. La dieta usada en la primera alimentación afecta la ganancia de peso y longitud total de larvas de cachama blanca (Piaractus brachypomus). Rev Colomb Cienc Pec. 2011; 24:48-53.

FAO - Organización de las Naciones Unidades para la Agricultura y la Alimentación. 2014. El estado mundial de la pesca y la acuicultura. Roma.
Landines PMA y Mojica BHO. 2005. Reproducción de peces en el trópico. MiniAgricultura - INCODER - UNAL. ISBN. 958-338515-8. pp 91 - 104.

Lazo J. 2000. Conocimiento actual y nuevas perspectivas en el desarrollo de dietas para larvas de peces marinos. Memorias del V Simposio Internacional de Nutrición Acuícola. Mérida, Yucatán, México, 300-312.

Merino MC, Bonilla AP, Bages F. 2013. Diagnóstico del estado de la Acuicultura en Colombia. Min Agricultura, FAO. ISBN: 978958-57974-0-6. pp 51-58.

Mesa M, Botero M. La cachama blanca (Piaractus brachypomus), una especie potencial para el mejoramiento genético. Rev Colomb Cienc Pec. 2007;20(1):79-86.

Morales LKA, Ortiz GAR, León MCA, Rodríguez PJA. 2014. Contribución al estudio de la resistencia y supervivencia a fluctuaciones térmicas en Poecilia reticulatas Peterm 1859. Memorias VI Congreso Colombiano de acuicultura. ISSN: 2389-959X. Universidad de los Llanos, Villavicencio - Colombia.

Moyle PB, Cech JJ. 2004. Fishes an Intoduction to Ichthyology. $5^{\mathrm{a}}$ Edition, Prentice Hall. $84-85$ pp.

Navarro OJ, Velasco SY, Cruz CPE. Evaluación de cinco protectores para la crioconservación de semen de Cachama Blanca (Piaractus brachypomus). Rev Col Cienc Pec. 2004;17(4):53-59.

Osse W M, Van Den Boogaart JG. Fish larvae, development, allometric growth and aquatic environment. ICES. Mar Sci Symp. 1995; 201:21-34.

Parker N, Davis K. 1981. Requirements of warm water fish. In: L. Allen and E. Kinney eds. Proc Bioengineering Sym Fish Culture. Fish Cul Sec Am Fish Soc, Bethesda, Maryland, USA. 21-28 pp.

Peñuela HZ, Hernández AG, Corredor MJR, Cruz CPE. Consumo de oxígeno en cachama blanca (Piaractus brachypomus) durante diferentes etapas de desarrollo corporal. Orinoquia. 2007;11(1):49-55.

Vásquez TW. "Crescimento de juvenis de Piaractus brachypomus alimentados com dietas contendo diferentes perfis de aminoácidos essenciais" En:Brasil Pesquisa Agropecuária Brasileira ISSN: 0100-204X ed: Embrapa 2013;48(8):849-856.

Vásquez TW, Zacarías SG. Aspectos reproductivos de la cachama blanca Piaractus brachypomus (Cuvier, 1818) I. Histomorfología de los ovocitos durante la ovogénesis. Rev ACOVEZ. 1996;21(3):18-24.

Vásquez TW, Pereira FM, Arias CJA. "Estudos para Composição de uma Dieta Referência Semipurificada para Avaliação de Exigências Nutricionais em Juvenis de Pirapitinga, Piaractus brachypomus (CUVIER 1818)". En: Brasil Revista Brasileira De Zootecnia-Brazilian Journal Of Animal Science ISSN: 1516-3598 ed: Sociedade Brasileira De Zootecnia. 2002;31(12):283-292.

Yossa PMI, Hernández AG. Vásquez TW y Ortega RJP. 2011. Informe técnico Proyecto Correlación entre la dinámica de nutrientes y la calidad del agua en estanques de cultivo comercial de tilapia roja (Oreochromis spp) y cachama blanca (Piaractus brachypomus). Convenio 0057/07 MADR - Unillanos. 